\title{
Exploiting Spatial and Frequency Diversity in Spatially Correlated MU-MIMO Downlink Channels
}

\author{
Rosdiadee Nordin \\ Department of Electrical, Electronics and System Engineering, Faculty of Engineering and Built Environment, Universiti Kebangsaan \\ Malaysia, 43600 Bangi, Selangor, Malaysia \\ Correspondence should be addressed to Rosdiadee Nordin, adee@eng.ukm.my
}

Received 23 April 2012; Revised 9 November 2012; Accepted 10 November 2012

Academic Editor: Rui Zhang

Copyright ( $) 2012$ Rosdiadee Nordin. This is an open access article distributed under the Creative Commons Attribution License, which permits unrestricted use, distribution, and reproduction in any medium, provided the original work is properly cited.

The effect of self-interference due to the increase of spatial correlation in a MIMO channel has become one of the limiting factors towards the implementation of future network downlink transmissions. This paper aims to reduce the effect of self-interference in a downlink multiuser- (MU-) MIMO transmission by exploiting the available spatial and frequency diversity. The subcarrier allocation scheme can exploit the frequency diversity to determine the self-interference from the ESINR metric, while the spatial diversity can be exploited by introducing the partial feedback scheme, which offers knowledge of the channel condition to the base station and further reduces the effect before the allocation process takes place. The results have shown that the proposed downlink transmission scheme offers robust bit error rate (BER) performance, even when simulated in a fully correlated channel, without imposing higher feedback requirements on the base controller.

\section{Introduction}

Dynamic resource assignment from the Orthogonal Frequency Division Multiple Access (OFDMA) in combination with multiplicative increase in throughput from MultipleInput Multiple-Output (MIMO) technology offers improved spectral diversity in a wireless downlink transmission. The result of this combination is able to provide a highly efficient and low latency with enhanced spectrum flexibility radio interface, as can be seen from the downlink implementation of a Long Term Evolution (LTE) network [1]. In addition, the LTE network benefits from MU-MIMO, a multiuser diversity technique that exploits the spatial diversity from the channel knowledge at the transmitter, that is, channel state information (CSI), to improve the performance gain. However, accurate CSI is obtained at the expense of massive feedback overhead. A partial feedback scheme, which is based on a quantized discrete Fourier transform (DFT), is considered in this paper. Instead of feeding back the full CSI, mobile users update the E-UTRAN Node B (eNodeB) with the preferred precoding matrix based on the channel quality indicator (CQI).
The implementation of the full feedback scheme comes at the expense of CSI; therefore, it requires an enormous amount of feedback to the eNodeB. This scenario is not practical for the downlink implementation because eNodeB requires a higher level of computational overhead to compute the channel matrix. This situation worsens when the channel is severely impaired by channel imperfection, such as spatial correlation, which is also described by Gesbert et al. [2] as an effect of self-interference. This is because MIMO system capacity mostly depends on the spatial correlation properties of the radio channel. An obvious way to achieve decorrelation between a set of antenna elements is to place them far away from each other. However, in most cases, the nature of the equipment will limit the antenna spacing.

The core novelty of this paper lies in the fact that it considers the effective exploitation of both the frequency and spatial diversity. The spatial diversity is exploited from the MU-MIMO feedback scheme, while the frequency diversity is implemented by means of dynamic subcarrier allocation. Both of the diversity schemes, spatial diversity (from MU-MIMO feedback scheme) and frequency diversity (dynamic subcarrier allocation), are combined in order to 
reduce the effect of self-interference at the user equipment (UE) and/or eNodeB (base station). In addition, two feedback schemes are considered: (i) full feedback, which has full information of the channel's CQI and (ii) partial feedback, which represents the channel's CQI in a quantized form. The partial feedback scheme aims to reduce the uplink overhead requirement. The proposed combination of diversity schemes is analyzed against different MIMOcorrelated channel environments and codebook sizes to achieve varying degrees of multiuser diversity.

The rest of this paper is organized as follows. Section 2 describes the fundamentals of MU-MIMO, Section 3 presents different types of feedback schemes, Section 4 presents the subcarrier allocation scheme, Section 5 describes the system setup and simulation parameters for the representation of the LTE downlink transmission, Section 6 discusses and analyses the significance of the results from the simulation, and Section 7 concludes the paper with a brief discussion.

\section{Spatial Diversity: Multiuser- (MU-) MIMO Scheme}

MIMO operation can be classified into two modes: Singleuser- (SU-) MIMO and Multiuser- (MU-) MIMO. SUMIMO only considers access to multiple antennas that are connected to a single UE. However, this configuration does not achieve ideal channel capacity when the channel is highly correlated and thus requires decorrelation between the spatial signatures of the antennas. In an Line-of-Sight LOS situation, a strong correlation between spatial signatures is expected. This correlation limits the use of spatial multiplexing and degrades the overall system throughput.

By scheduling different UEs on different spatial streams over the same time-frequency resource, additional diversity can be exploited in the spatial domain. This spatial diversity scheme is called MU-MIMO. MU-MIMO allows the simultaneous allocation of different spatial subchannels to different UEs in the same time-frequency resource. The earlier work in MU-MIMO can be traced back in 2006, published by Weingarten et al. [3].

In MU-MIMO, the burden of spatially separating the UEs lies at the eNodeB, thus offering reduced complexity at the UE compared to SU-MIMO. MU-MIMO benefits from the knowledge of channel state information (CSI) to properly serve the spatially multiplexed users. CSI, while not essential to SU-MIMO, is of critical importance to downlink MUMIMO precoding techniques.

\section{Feedback Schemes in MU-MIMO}

Precoding uses a linear transformation of the symbols at the transmitter to improve the resilience of spatial multiplexing. In a precoded system, CSI allows a transmitter to send data along the strongest eigenmodes of a channel and effectively cope with fading by appropriate weighting from the beamforming.
However, the use of precoding comes at the expense of channel knowledge. Precoding requires an enormous amount of feedback on the uplink to benefit most from the full channel information. Therefore, full and accurate CSI feedback may not be available in a severely impaired channel, reducing the overall spectral efficiency as a result of the quantization error. These challenges have motivated research into the partial feedback scheme.

3.1. Partial Feedback versus Full Feedback. In the full feedback scheme, a UE feeds back a channel quality indicator (CQI) value for every matrix in the codebook, as is illustrated in Figure 1. When a precoding matrix for the RB is chosen, the corresponding SINR can be fed into the scheduler, which provides accurate CQI information. The user with the highest SINR for each spatial subchannel is selected, and the selected users are then precoded to share the same timefrequency resource grid.

While in a partial feedback scheme, the transmitter is provided with quantized information, and most of the gains of a precoded spatial multiplexing system can be achieved at the cost of a few bits of feedback. For example, Sanayei and Nosratinia [4] have shown that a single bit quantized feedback can preserve the multiuser diversity gain. A user selects one or more preferred beamforming matrices out of the codebook by evaluating the SINRs of different beamforming combinations. Thus, each user must signal one or several indices of the preferred vectors. The UE only feeds back a single CQI value for the preferred precoding matrix for each RB, by selecting the highest average SINR perceived by the user. Based on the feedback, the scheduler at the eNodeB chooses the precoding matrix with the highest sum of the average SINR values of all spatial subchannels and applies it to the RB.

In the full feedback scheme, the corresponding SINR is known to the scheduler because the eNodeB has the information from all users. While in the partial feedback scheme, only the users who declare the same preferred matrix are eligible for selection. For MU-MIMO, feedback schemes must be jointly designed with appropriate scheduling and multiple access methods to minimize the CSI inaccuracy and retain the feedback efficiency.

3.2. Unitary Precoding. In unitary precoding (UP), UEs provide accurate and efficient CSI feedback regarding the preferred precoding matrix to the eNodeB on the uplink control channel. Ideally, this information is made available per resource block, thus allowing channel resources to be allocated to different users in an effective manner and allowing the amount of feedback to be greatly reduced. There are several precoding methods (references $[5,6]$ provide detailed overview of precoding scheme with limited feedback) that have been developed, and one of them, known as the codebook-based precoding method, has recently received considerable attention in the literature.

A codebook consists of a finite number of possible beamforming matrices at both the transmitter and the receiver. Instead of using the physical antenna, the network 

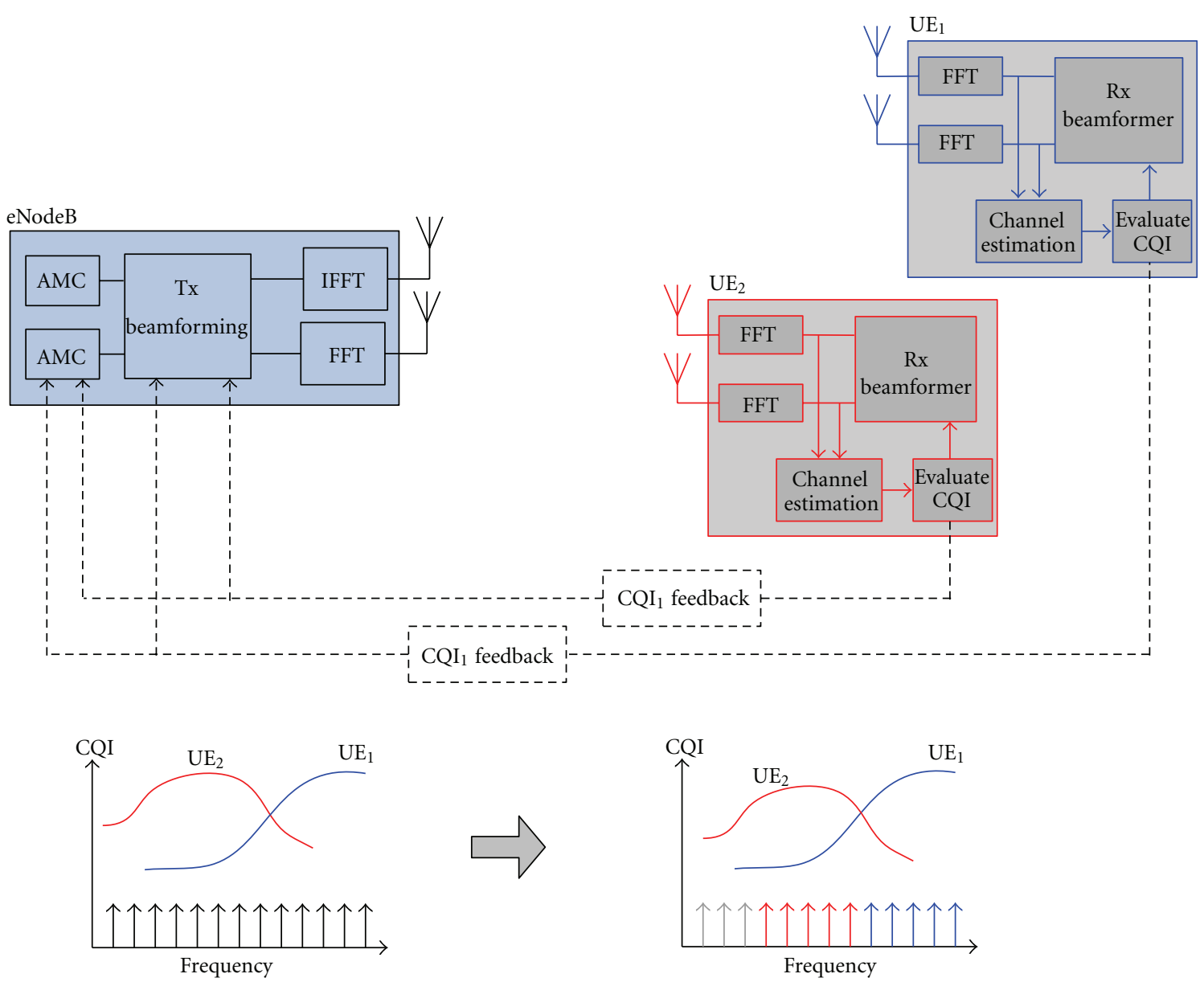

FIgURE 1: MU-MIMO with CQI feedback.

transmits through a codebook-based spatial beam, thus ensuring uniform sector coverage across the cell, as shown by Rohling and Grünheid [7].

This paper considers the use of DFT-based codebooks because it is shown in [8-10] that the DFT-based codebook is effective against a wide range of propagation scenarios, from the uncorrelated $[8,10]$ to the fully correlated channel [9]. Other than its excellent protection against the effects of self-interference, the DFT-based codebook also has low complexity codebook design, as described in [5].

The DFT-based unitary precoder codebook, E, consists of the unitary matrix set, that is, $\mathbf{E}=\left\{\mathbf{E}^{(0)} \cdots \mathbf{E}^{(l-1)}\right\}$, where $\mathbf{E}^{(l)}=\left[e_{0}^{(l)} \cdots e_{N_{t}-1}^{(l)}\right]$ is the $l$ th precoding matrix and $e_{n_{t}}^{(l)}$ is the $N_{t}$ th precoding vector in the set. It is defined in the Fourier basis, as given in [11]:

$$
\begin{aligned}
& e_{n_{t}}^{(l)}=\frac{1}{\sqrt{N_{t}}}\left[w_{0 n_{t}}^{(l)} \cdots w_{\left(N_{t}-1\right) n_{t}}^{(l)}\right]^{T}, \\
& w_{n_{r} n_{t}}^{(l)}=\exp \left\{j \frac{2 \pi n_{r}}{N_{t}}\left(n_{t}+\frac{l}{L}\right)\right\},
\end{aligned}
$$

where $N_{t}$ and $N_{r}$ are the number of receiving and transmitting antennas, respectively; $w_{m n}^{(l)}$ is the codebook index and $L$ is the codebook size. From (1), the precoder matrices sets
TABLE 1: Codebook size for unitary precoding, $L=2$.

$$
\begin{aligned}
& \mathbf{E}_{0}=\frac{1}{\sqrt{2}}\left[\begin{array}{cc}
1 & 1 \\
1 & -1
\end{array}\right] \\
& \mathbf{E}_{1}=\frac{1}{\sqrt{2}}\left[\begin{array}{cc}
1 & 1 \\
j & -j
\end{array}\right]
\end{aligned}
$$

(also known as the codebooks) of size 2 and 4 are generated; they are shown in Tables 1 and 2.

In codebook unitary precoding, the codebook size contains a set of $L=N_{q} / N_{t}$ predefined and fixed unitary beamforming matrices of size $N_{t} \times N_{t}$, where $N_{q}$ is the allocated subcarriers at spatial subchannel $q$. The codebook indices are then used by the eNodeB to construct the precoding matrix. For each beamforming matrix in the codebook, each UE computes the SINR for each of the $N_{t}$ beamforming vectors in the matrix assuming that the other spatial subchannels defined by the remaining $N_{t}-1$ vectors are used for interfering transmission to the other UEs.

Overall, the UE computes $N_{q}$ SINRs and signals the codebook index corresponding to the best SINR value 
TABLE 2: Codebook size for unitary precoding, $L=4$.

$$
\begin{gathered}
\mathbf{E}_{0}=\frac{1}{\sqrt{2}}\left[\begin{array}{cc}
1 & 1 \\
1 & -1
\end{array}\right] \\
\mathbf{E}_{1}=\frac{1}{\sqrt{2}}\left[\begin{array}{cc}
1 & 1 \\
\frac{1}{\sqrt{2}}(1+j) & \frac{1}{\sqrt{2}}(-1-j)
\end{array}\right] \\
\mathbf{E}_{2}=\frac{1}{\sqrt{2}}\left[\begin{array}{cc}
1 & 1 \\
j & -j
\end{array}\right] \\
\mathbf{E}_{3}=\frac{1}{\sqrt{2}}\left[\begin{array}{cc}
1 & 1 \\
\frac{1}{\sqrt{2}}(-1+j) & \frac{1}{\sqrt{2}}(1-j)
\end{array}\right]
\end{gathered}
$$

back to the eNodeB. The eNodeB then makes use of this information to select the beamforming matrix and schedule the UEs for transmission. In a partial feedback scheme, the eNodeB has a very limited set of unitary precoding matrices from which to choose, and the multiplexing gain is at its maximum when enough UEs in the cell have orthogonal channel signatures that match the vectors in one of the codebook matrices.

For both the MU-MIMO and SU-MIMO cases, the amount of feedback increases with the number of spatial subchannels, $Q$. In the full feedback MU-MIMO scheme, the amount of feedback is further increased by the codebook size (an $L$ fold increase), resulting in a further increase in the uplink overhead. Otherwise, the same precoding matrix is shared among all of the subcarriers in an RB. The feedback overhead for each RB for the considered MIMO scheme is summarized in Table 3 . The reporting scheme for MUMIMO scheme is based on CQI per layer. A bitmap (e.g., 4 bits) is used to signal the MCS levels. The preferred antennas are implied by filling in the appropriate CQI layer field. The number of " $1 \mathrm{~s}$ " in the bitmap indicates the rank for antenna selection, while a NULL CQI field indicates the corresponding transmit antenna is not preferred. The matrix index represents the preferred DFT matrix by indicating " 1 " in the corresponding bit position.

\section{Frequency Diversity: Interference-Aware Subcarrier Allocation}

4.1. SINR Metric. The mathematical model of a received signal in the considered MIMO-OFDMA system, after FFT and guard removal, is described as follows:

$$
\mathbf{Y}_{k}^{s}=\mathbf{H}_{k}^{s} \mathbf{E}_{k}^{s} \mathbf{X}_{k}^{s}+\mathbf{N}_{k}^{s},
$$

where the subscript $k$ denotes the UE index, $s$ denotes the subcarrier index, $\mathbf{H}_{k}^{s}$ is a channel matrix containing the frequency responses of the spatial subchannels between $N_{t}$ and $N_{r}$ antennas at subcarrier $s$ and applied to the subcarriers of the OFDMA signal on a cluster basis for the $k$ th $\mathrm{UE}, \mathbf{E}_{k}^{s}$ is the precoding matrix, $\mathbf{N}_{k}^{s}$ denotes a complex circular symmetric colored noise with an invertible
TABLE 3: Feedback overhead for the considered MIMO schemes for $L=2, Q=2$.

\begin{tabular}{lccc}
\hline Feedback Scheme & $\begin{array}{c}\text { MU-MIMO } \\
\text { full } \\
\text { feedback }\end{array}$ & $\begin{array}{c}\text { MU-MIMO } \\
\text { partial } \\
\text { feedback }\end{array}$ & SU-MIMO \\
\hline Preferred layer 1 CQI & 4 bits & 4 bits & 4 bits \\
Preferred layer 2 CQI & 4 bits & 4 bits & \\
Alternative layer 1 CQI & 4 bits & - & - \\
Alternative layer 2 CQI & 4 bits & - & - \\
Preferred matrix index & 1 bit & 1 bit & 1 bit \\
\hline Total bits per RB & 17 bits & 9 bits & 5 bits \\
\hline
\end{tabular}

covariance matrix, and $\mathbf{X}_{k}^{s}$ denotes the $N_{t} \times 1$ matrix containing the transmitted signals.

At the receiver, the proposed MIMO detection adopts an MMSE linear receiver:

$$
\mathbf{G}_{k}^{s}=\left(\left(\mathbf{E}_{k}^{s}\right)^{\mathbf{H}}\left(\mathbf{H}_{k}^{s}\right)^{\mathbf{H}} \mathbf{H}_{k}^{s} \mathbf{E}_{k}^{s}+Q \frac{\mathbf{N}_{k}^{s}}{\varepsilon} \mathbf{I}\right)^{-1}\left(\mathbf{E}_{k}^{s}\right)^{\mathbf{H}}\left(\mathbf{H}_{k}^{s}\right)^{\mathbf{H}},
$$

where $Q$ is the number of data streams and $\varepsilon$ is the transmit symbol energy. The number of data streams in this work is assumed to be limited by $\min \left(N_{r}, N_{t}\right)$. In the case of SU-MIMO, both spatial streams go to the same UE. The MMSE filter is chosen because it has the ability to mitigate self-interference without adversely amplifying the received noise. The MMSE filter is also able to separate the spatial subchannel of the MIMO structure, as shown by Jang and Lee [12]. The received signal is multiplied by the MMSE filter (3) to obtain the detected data stream, $\mathbf{D}_{k}^{s}$ :

$$
\mathbf{D}_{k}^{s}=\mathbf{G}_{k}^{s} * \mathbf{Y}_{k}^{s}=\hat{\mathbf{X}}_{k}^{s}+\mathbf{N}_{k}^{s} .
$$

For each data stream $q$ at each RB, UE $k$ then computes the SINR for every subcarrier (the subcarrier index $s$ is omitted for ease of reference):

$$
\begin{aligned}
& \mathbf{S I N R}_{k}^{q} \\
& =\frac{\left|\left(\mathbf{G}_{k} \mathbf{E}_{k} \mathbf{H}_{k}\right)_{q q}\right|^{2} \varepsilon}{\left|\left(\mathbf{G}_{k} \mathbf{E}_{k} \mathbf{H}_{k}\right)_{q j, j \neq q}\right|^{2} E_{s}+\left(\left|\mathbf{G}_{k}\right|_{q q}^{2}+\sum_{j \neq q}\left|\mathbf{G}_{k}\right|_{q j, j \neq q}^{2}\right) \mathbf{N}_{k}^{s}},
\end{aligned}
$$

where $q$ is the spatial subchannel at every subcarrier and $|X|_{q j}$ denotes the element located in row $q$ and column $j$ of matrix $X$. The SINR metric aims to compute the selfinterference from the data stream component $|Y|_{q q}$ and the self-interference component $|Y|_{q j, j \neq q}$ from the other transmitted data streams within the same subchannel. In this work, it is proposed that the allocation will be based on the sum of the achievable capacity of both spatial streams.

In an SU-MIMO system, for RB $c$, denoting the index of starting subcarrier by $n$ and the finishing subcarrier by $m$, the average rate of user $k$ is given by

$$
r_{k, c}=\frac{1}{m-n+1} \sum_{s=n}^{m} \sum_{q}^{\min \left(N_{t}, N_{r}\right)} \log _{2}\left(1+\operatorname{SINR}_{k, s}^{q}\right) .
$$


The eNodeB allocates each RB to user according to the selected resource allocation algorithm. The scheduler then uses this feedback information to allocate the RB to the UE with the highest achievable data rate, $r_{k}^{q}$. Compared to SU-MIMO, the scheduling for the decision to choose the best precoding matrix is slightly complicated in MUMIMO. To maximize the system capacity of MU-MIMO, the most suitable precoding matrix must be selected from the codebook to transmit on each RB. Based on the chosen precoding matrix, a dynamic subcarrier allocation is employed to select the user with the best channel condition. The selected users are then precoded to share the same time and frequency resources to maximize the system capacity.

Because each of the spatial streams can be allocated and scheduled independently in MU-MIMO, the UE $k$ calculates the data rate of each spatial layer and feeds it back to the BS. The user $k$ calculates the data rate of each spatial layer $q$ on a RB basis.

$$
r_{k, s}^{q}=\frac{1}{m-n+1} \sum_{s=n}^{m} \log _{2}\left(1+\operatorname{SINR}_{k, s}^{q}\right) .
$$

Again, for every RB, the scheduler allocates each spatial layer to the UE that has the best channel conditions for the corresponding layer.

4.2. Subcarrier Allocation. The interference-aware subcarrier allocation scheme uses the SINR as the performance metric to determine the allocation. The SINR metric has the knowledge of a particular subcarrier that is affected by selfinterference, especially when the correlation is high inside the spatial subchannels.

The allocation ranks users from the lowest to highest SINR metric in each spatial subchannel. Consequently, the next best subcarriers are allocated to users in rank order, allowing users with the lowest SINR at that particular spatial subchannel to have the best SINR that is available for the next transmission. Each MS provides SINR information to the BS, and the subcarrier allocation algorithm then allocates subcarriers to the MSs.

The nomenclatures are set first as references. In the following algorithm, $q=\{1, \ldots, Q\}$ represents the effective spatial subchannel considered for the allocation algorithm. $\Sigma_{k}^{q}$ represents the average SINR metric for user $k$ in the $q$ th spatial subchannel, $K$ is the total number of users, and $S$ is a $Q$ by $N_{q}$ matrix in which each row is a vector containing the indices of the useable subcarriers for the particular spatial subchannel (i.e., $N_{q}=\left\{1, \ldots, N_{\text {sub }}\right\}$, where $N_{\text {sub }}$ is the total number of useable subcarriers). $\operatorname{SINR}_{k, s}^{q}$ is the SINR matrix for user $k$ at subcarrier $s$ and spatial subchannel $q$, and $\mathrm{C}_{s, k}$ is a matrix that stores the subcarrier indices (subcarrier location) of the allocated subcarriers for user $k$ and subcarrier $s$.

The following algorithm performs the proposed interference-aware subcarrier allocation.

(1) After the eNodeB transmits the data matrix $\mathbf{X}_{k}^{s}$, the $k$ th UE computes the MMSE filter (3).

(2) The $k$ th mobile station then computes the SINR (5) of the $q$ th spatial subchannel.
(3) With the feedback information from UE, eNodeB allows the user with the lowest data rate to have the next choice of best subcarrier as follows.

(a) Generate short list of users and start with the user with the least SINR, $\boldsymbol{\Sigma}$ (For the first iteration in the allocation scheme, when no subcarriers have been allocated, all users assumed to have equal SINR value; thus the list appears entirely arbitrary). Find user $k$ satisfying

$$
\Sigma_{k}^{q} \leq \Sigma_{i}^{q} \quad \forall i, 1 \leq i \leq K .
$$

(b) For the user $k$ in (a), find the subcarrier $s$ satisfying

$$
\operatorname{SINR}_{k}^{q} \geq \operatorname{SINR}_{j}^{q} \quad \forall j \in N .
$$

(c) Update $\operatorname{SINR}_{k}^{q}$, $N_{q}$, and $\mathbf{C}_{s, k}$ with $k$ and $s$ in (b) according to

$$
\begin{gathered}
\Sigma_{k}^{q}=\boldsymbol{\Sigma}_{k}^{q}+\operatorname{SINR}_{k, s}^{q}, \\
N_{q}=N_{q}-s, \\
\mathbf{C}_{s, k}=s,
\end{gathered}
$$

where $N_{q}$ is the allocated subcarriers at spatial subchannel $q$ and $\mathbf{C}_{s, k}$ is the allocation matrix to record the allocated subcarrier $s$ for user $k$.

(d) Go to the next user in the short list in (a) until all users are allocated another subcarrier, $N \neq 0$ in $(\mathrm{c})$.

In this algorithm, users are not allowed to share subcarriers, thus reducing the complexity of the algorithm. In the SISO case, Jang and Lee [12] show that the capacity can be maximized if a subcarrier is only assigned to one user because the interference from other users' signals that share the same subcarrier is reduced. This paper extends the theory presented in [12] into the MIMO case, in which the number of users sharing the same subcarrier is limited to the number of available spatial subchannels (two subchannels in this case).

\section{Simulation Environment and Parameters}

This paper aims to apply the feedback schemes to spatially correlated subchannel environments. In an MU-MIMO downlink environment, the effect of self-interference must be considered because the spatial correlation experienced by the UE in a real channel environment can vary from an ideally uncorrelated channel to a fully correlated channel.

This study considers an urban microenvironment with a $500 \mathrm{~m}$ cell radius, outdoor terminals, and $2 \mathrm{GHz}$ frequency band. This environment is represented by the 3GPP-SCM Urban Micromodel [13], with an RMS delay spread of $251 \mathrm{~ns}$, an excess delay of $923 \mathrm{~ns}$, and a Nonline-of-Sight (NLOS) propagation scenario. The total number of available subcarriers depends on the overall transmission bandwidth 
TABLE 4: OFDMA parameters.

\begin{tabular}{lc}
\hline Parameters & Value \\
\hline Downlink bandwidth & $10 \mathrm{MHz}$ \\
Time slot/subframe duration & $0.5 \mathrm{~ms} / 1 \mathrm{~ms}$ \\
Subcarrier spacing & $15 \mathrm{kHz}$ \\
Precoding codebook size, $L$ & 2 \\
FFT size, $N_{\text {FFT }}$ & 1024 \\
Useable subcarrier, $N_{\text {sub }}$ & 600 \\
Number of OFDM symbols per time slot & $7 / 6$ \\
(Short/Long CP) & \\
\hline
\end{tabular}

TABLE 5: Correlation scenarios.

\begin{tabular}{lcc}
\hline Correlation modes & $R_{\mathrm{MS}}$ & $R_{\mathrm{BS}}$ \\
\hline Uncorrelated & 0.00 & 0.00 \\
Fully correlated & 0.99 & 0.99 \\
\hline
\end{tabular}

of the system. For this simulation, the representations of the LTE-OFDMA downlink parameters are summarized in Table 4.

The proposed interference-aware subcarrier allocation is simulated in QPSK, with a $1 / 2$ rate modulation and coding scheme (MCS). This combination is one of the available MCS, as specified in [1]. QPSK modulation is chosen in the simulation because of its robustness and its ability to tolerate higher levels of self-interference, which makes it suitable for transmission in lower SNR region at the expense of lower transmission bit rate.

The performance of the subcarrier allocation in combination with the partial feedback scheme will be simulated against different spatial correlation MIMO environment, that is, self-interference effect. Therefore, the Urban Microchannel model is further extended into two correlated channel environments: (i) an uncorrelated channel that represents ideal channel conditions, in which the effect of self-interference is minimal and (ii) a "fully" correlated channel, which represents a worst case spatial correlation scenario. In a fully correlated channel, the effective MIMO channel is similar to a SISO system. The spatial correlation matrix of the MIMO channel, $\mathbf{R}_{\text {MIMO, is the Kronecker }}$ product of the spatial correlation matrix at the BS and the MS, $\mathbf{R}_{\text {MIMO }}=\mathbf{R}_{\text {MS }} \otimes \mathbf{R}_{\mathrm{BS}}$, as proposed by Beh et al. [14]. The proposed correlation scenarios are summarized in Table 5.

Three different feedback schemes are considered: (i) full feedback, (ii) partial feedback (both of these are applied to the MU-MIMO case) and (iii) SU-MIMO, which represents a case without any form of feedback. A set of 2000 independent identically distributed (i.i.d.) quasi-static Rayleigh distributed time samples per user are used in the simulation. A single CQI, which is based on the average SINR of the 12 grouped subcarriers, is fed back for each resource block (RB), and it is assumed to be perfectly known. The result is simulated based on 10 users, unless otherwise stated. A $2 \times 2$ antenna configuration is considered for the simulation. Because of the increased computational complexity and the insignificant power control gain in the frequency domain dynamic allocation, equal power allocation is assumed throughout the simulations.

\section{Error Performance Analysis}

6.1. Comparison between Different Feedback Schemes. The proposed interference-aware subcarrier allocation scheme is compared against another subcarrier allocation scheme, known as the Dynamic Subcarrier Allocation (DSA), as proposed in [15]. In the DSA scheme, the subcarrier allocation at each spatial layer is treated independently, and channel gain is used as the performance metric to determine the subcarrier allocation.

Figure 2 illustrates the BER performance in both the uncorrelated and fully correlated channel. MU-MIMO shows significant improvement over SU-MIMO; the margin of difference is approximately $4 \mathrm{~dB}$ at $\mathrm{BER}=10^{-3}$. This result confirms the multiuser benefit that MU-MIMO can offer. For the SU-MIMO case, the poor performance can be explained by the spatial correlation coefficient $\left(R_{\mathrm{MIMO}}=\right.$ 0.99); in this case, the effective channel in the SU-MIMO system is similar to a SISO (i.e., a single data stream). The increase in the BER gain between MU-MIMO and SU-MIMO is significant, especially at higher SNR. The DSA offers better performance when simulated in the uncorrelated channel; however, it suffers from severe BER loss as the channel achieves full correlation. This loss occurs because there is no knowledge of the channel condition from the subcarrier allocation scheme because channel gain is used as the performance metric. Consequently, the results from SU-MIMO are not considered in this paper.

The advantage of the interference aware allocation scheme is significant in a fully correlated channel, as illustrated in Figure 3. Full feedback MU-MIMO offers higher BER performance than SU-MIMO, for which the margin of difference is approximately $2.5 \mathrm{~dB}$ at $\mathrm{BER}=10^{-3}$ in a fully correlated channel. In the case of the MU-MIMO feedback schemes, full feedback offers superior BER improvement to partial feedback, for which the margin of difference is approximately $2 \mathrm{~dB}$ at $\mathrm{BER}=10^{-3}$.

Nevertheless, MU-MIMO has been shown to achieve more BER gain through the additional dimension of diversity in the spatial domain in both correlation environments by exploiting the spatial subchannel as an additional dimension for allocating resources. By utilizing the interference-aware allocation scheme at each spatial dimension, the effect of self-interference is further reduced. MU-MIMO also has a large wavelength separation between the UEs, thus allowing a higher degree of decorrelation.

6.2. Performance of Different Codebook Sizes. In this section, four codebook sizes are compared, $L=\{1,2,4,8\}$. In a fully correlated channel, the advantage of larger codebook sizes over smaller ones is obvious, particularly for the partial feedback scheme, as shown in Figure 4. However, in an uncorrelated channel, the BER performance for $L=8$ is only marginally better than that of $L=2$. This lack of improvement occurs as a result of the lower multiuser 


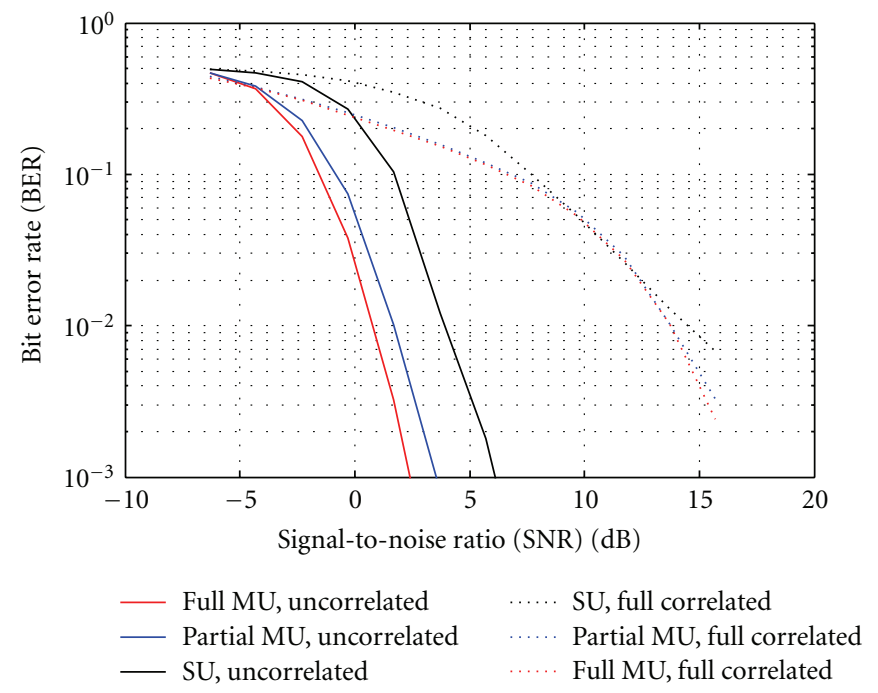

FIGURE 2: BER performance for DSA, for $L=2$ in different types of feedback.

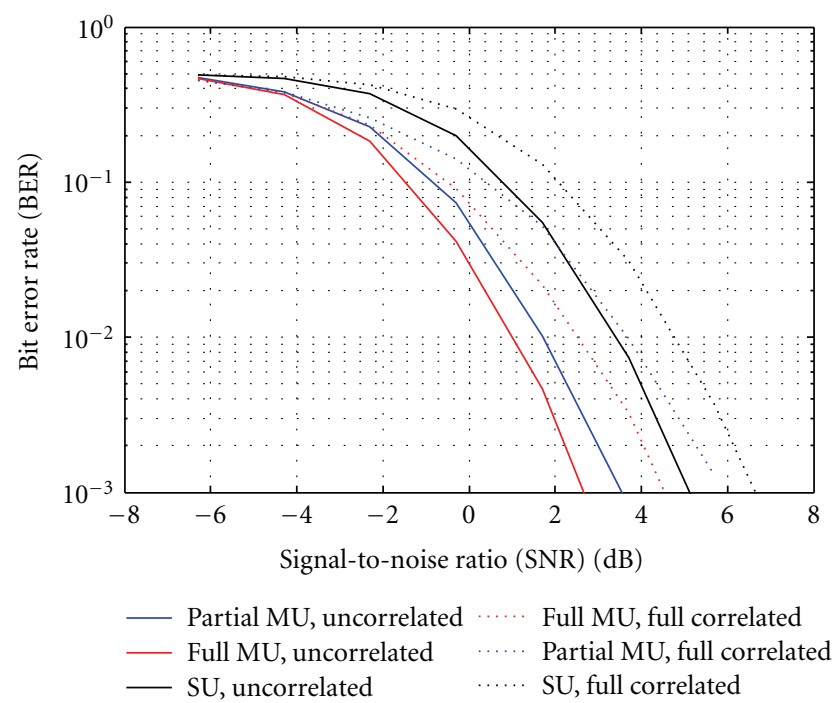

FIGURE 3: BER performance for interference-aware allocation, for $L=2$ with different types of feedback.

diversity gain that can be achieved by the partial feedback scheme because the feedback information has been limited at the scheduler. In the case of the full feedback scheme, a higher BER gain can be achieved by larger codebook sizes, as shown in Figure 5. As an example, the codebook with a size of $L=8$ achieved $3 \mathrm{~dB}$ gain over a codebook with a size of $L=2$ in a fully correlated channel at BER $=10^{-3}$.

From these results, it can be generally stated that a larger codebook size leads to better BER performance in the full feedback scheme, which helps to reduce the effect of selfinterference. This improvement occurs because of the greater selection of precoding matrices. A larger codebook contains more distinct codewords, which increases the possibility that each spatial subchannel can find a better codeword match during encoding. Thus, larger codebooks are more

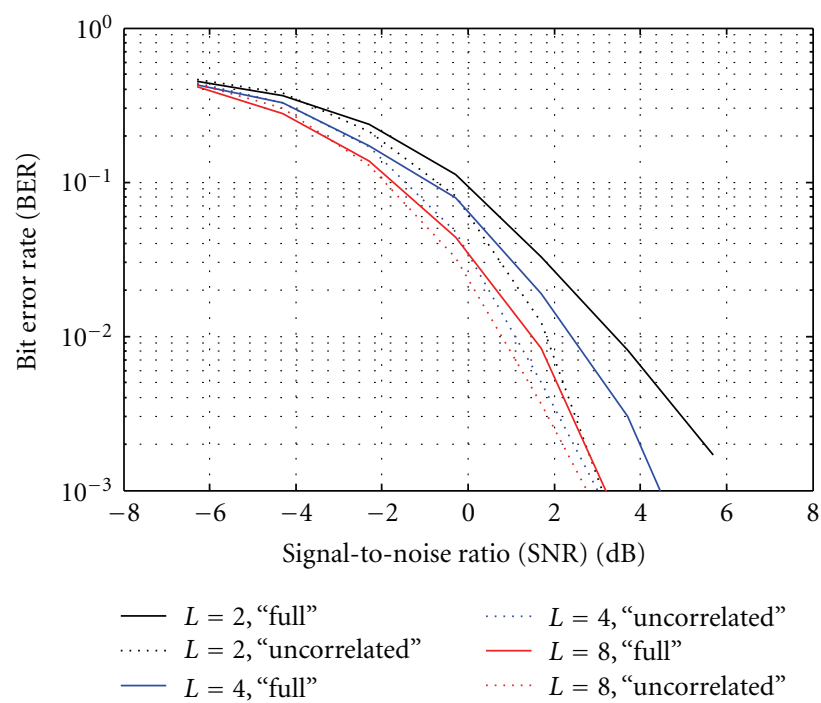

FIGURE 4: BER performance between different codebook sizes (partial feedback MU-MIMO).

likely to have a lower BER loss, allowing a more accurate representation of the channel condition. Further, the use of DFT-based codebook precoding effectively reduces the impact of self-interference in a highly correlated channel.

The results published by Ravindran and Jindal [16] also confirm these findings, showing that, in a fully correlated channel, the DFT-based codebook can reduce the channel information amplitude difference between different transmitting antennas, while a constant modulus DFT codebook produces less quantization error than nonconstant modulus Grassmannian-based codebook precoding. A recent publication by Yang et al. [9] also demonstrated that the DFT-based codebook is effective against spatial correlation in an MUMIMO system. 


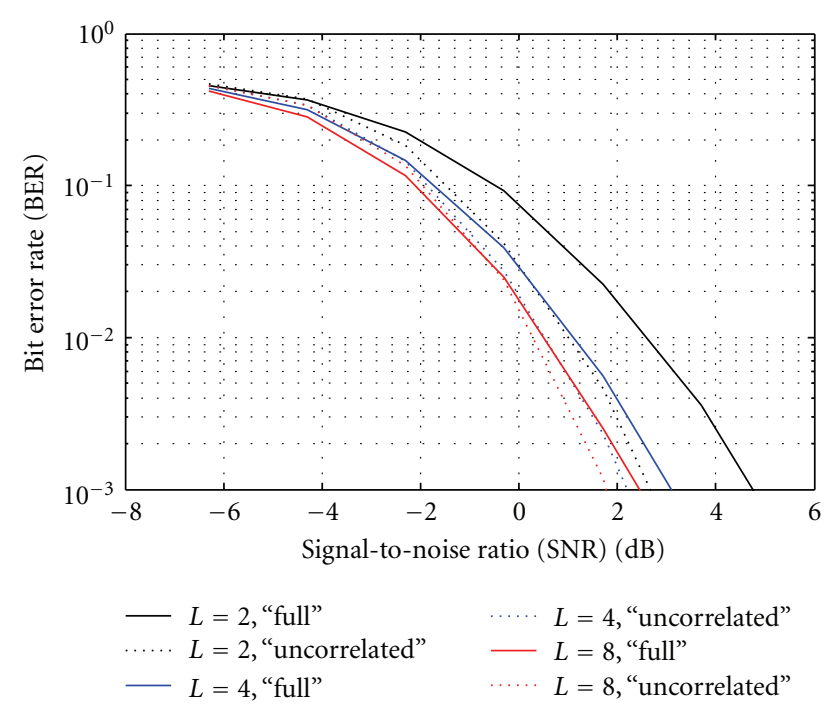

FIGURE 5: BER performance between different codebook sizes (full feedback MU-MIMO).

The BER performance of the MU-MIMO feedback schemes is expected to increase further with larger codebook size. However, using a codebook size that is too large can also pose a few issues. First, a larger codebook size decreases the spectral efficiency of the uplink channel because the feedback information increases considerably. Increasing the codebook size incurs exponential complexity in quantizing the CQI, as shown by Beh in [17], thus defeating the main purpose of channel feedback, particularly in the partial feedback scheme. At the time of writing, only a codebook size of $L=2$ is supported by the 3GPP specification published in [18] because it trades off between performance gain and feedback overhead.

It is also shown that in a fully correlated channel, larger codebook sizes ( $L \geq 4$ ) offer superior performance to small codebook size $(L=2)$. This result suggests that the size of the codebook has implications for the tradeoff between the numbers of signaling bits required to indicate a particular matrix in the codebook and the suitability of the resulting transmitted beam direction. It is shown by Tse and Viswanath [19] that the codebook size $L=4$ has a marginally better multiuser diversity gain than $L=2$ when simulated in the partial feedback scheme. This improvement is observed because the number of users selecting the same codebook matrix is reduced for the larger codebook size, causing a smaller number of users to be scheduled on the same timefrequency resource. For these reasons, it is important to select a suitable codebook size for the MU-MIMO downlink transmission.

6.3. Performance Comparison between Partial and Full Feedback Schemes. Figures 6 and 7 compares the BER performance between the partial feedback and full feedback schemes for different numbers of users in uncorrelated and fully correlated channels, respectively. As the number of users increases from 1 to 25 , the BER performance significantly

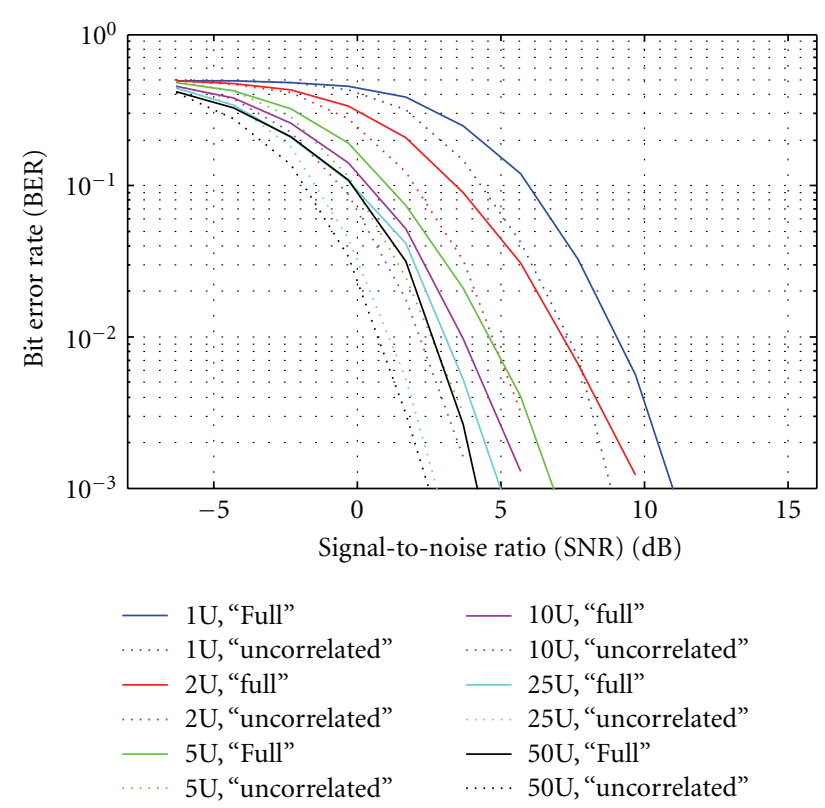

FIGURE 6: BER performance of an MU-MIMO (partial feedback) with different number of users.

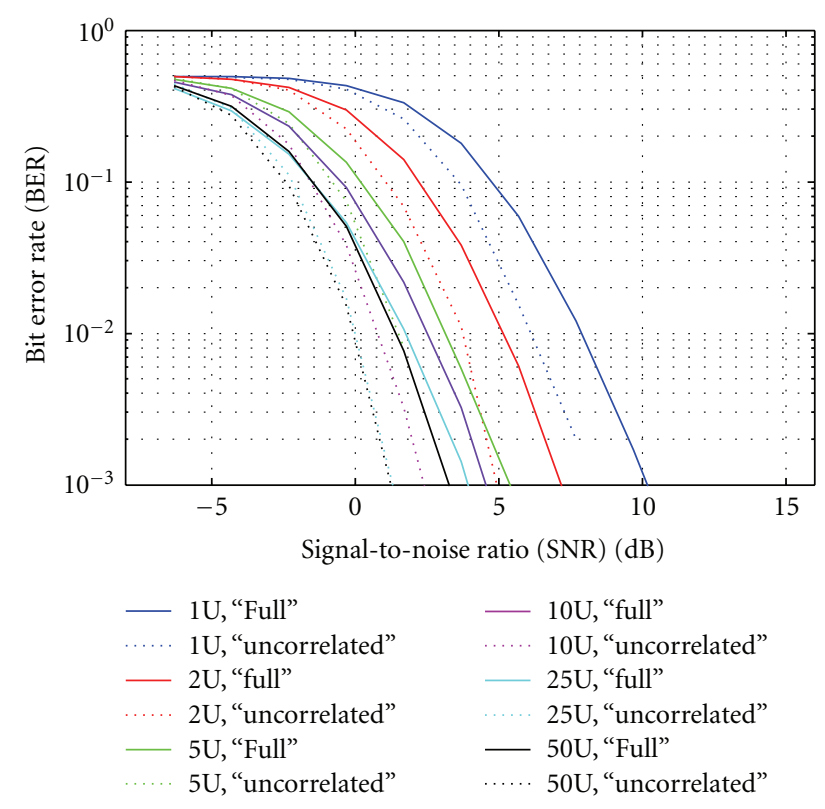

FIGURE 7: BER performance of an MU-MIMO (full feedback) with different number of users.

increases as a result of the richer spectral multiuser diversity gains. When the number of users is increased (e.g., from 10 to 50), the gain of multiuser diversity can be observed to increase slowly, suggesting that the multiuser diversity gain achieves saturation as the number of users increases. This result is consistent with theoretical observations made by Tse and Viswanath [17], in which greater gain can be achieved through the additional dimension of diversity in the spatial domain. MU-MIMO can achieve a similar level of diversity gain, even with fewer users in the system. 


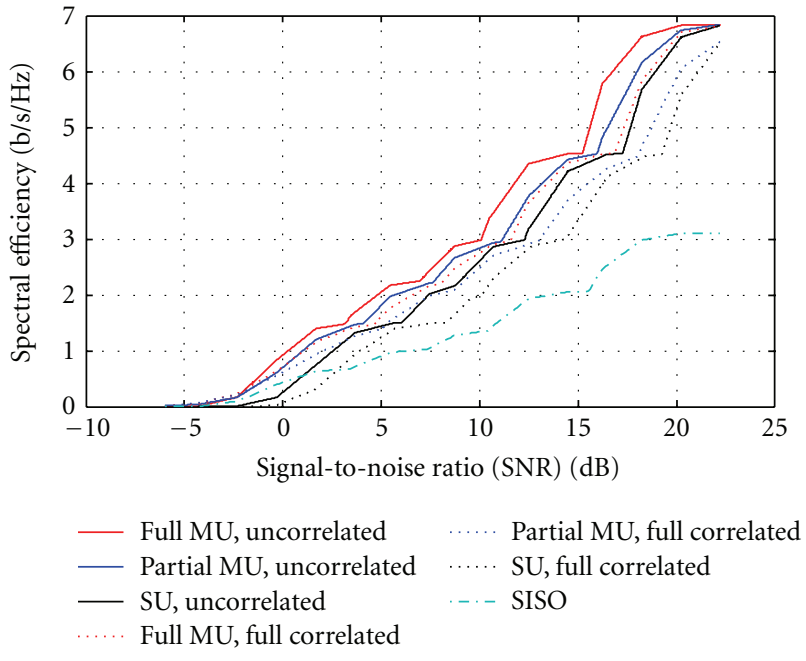

FIGURE 8: Average bandwidth efficiency comparison between the uncorrelated and fully correlated channels in the partial feedback scheme.

This result also confirms that, in addition to the ability to mitigate the effect of self-interference, the proposed interference-aware allocation scheme also considers the effect of multiple access interference (MAI) when simulated in MU-MIMO. In MU-MIMO, the resulting multiuser interference is handled by the multiple antennas, which provide the degrees of freedom necessary for spatial separation of the users, as well as link diversity. For a higher number of users in a full feedback scheme, a larger codebook size can further improve the result.

6.4. Throughput Analysis. Figure 8 compares the average bandwidth efficiency in uncorrelated and highly correlated channel environments. The bandwidth efficiency of a SISO system is plotted as a reference. The simulation result considers 10 users in both correlation cases.

From the figures, MIMO systems in the uncorrelated channel have better bandwidth efficiency than those in "fully" correlated channels. With the additional spatial diversity that can be exploited, MU-MIMO can provide almost double the throughput across the entire SNR range. In the case of "fully" correlated channels, MU-MIMO achieves significant bandwidth efficiency gain over SUMIMO. The partial feedback scheme is marginally inferior to the full feedback scheme in both uncorrelated and correlated channels.

At lower SNR, the bandwidth efficiency of MU-MIMO is similar to that of uncorrelated MU-MIMO and other feedback schemes because the use of QPSK modulation is robust against the effect of channel imperfection (especially channel correlation) in this case. At higher SNR, the higher level of MCS is vulnerable to channel degradation. By employing an adaptive modulation scheme, the proposed interference-aware allocation is also able to reduce the selfinterference effect across all SNRs.
Considering the correlation environment simulated in a fully correlated channel (correlation coefficient of 0.99), the proposed interference-aware allocation scheme is expected to offer higher throughput gain in practical applications based on the results presented in this paper.

\section{Conclusion}

In this paper, it is shown that the proposed interferenceaware allocation scheme (frequency diversity), in combination with the feedback schemes from MU-MIMO (spatial diversity), can improve the BER performance when simulated in a rapidly time-varying channel, which suffers from severe spatial correlation between the transmitting and receiving antennas.

There are two types of MU-MIMO feedback: (i) full feedback and (ii) partial feedback. Full feedback offers superior performance compared to partial feedback, but at the expense of high uplink overhead, while partial feedback offers a tradeoff between multiuser diversity gain and a reduced feedback requirement on the uplink.

In general, a larger codebook implies more accurate knowledge of the MIMO channel at the transmitter, which leads to improved diversity. There is no significant difference in BER performance between codebook sizes when the channel is uncorrelated and operating under single user (SUMIMO) transmission. The DFT-based codebook adaptation enables the quantization to exploit the spatial correlation inherent in the channel.

These results show that the combination of a codebook size of $L=2$ with the partial feedback scheme achieves marginal BER performance compared to higher $L$ sizes with full feedback scheme. The utilization of dynamic subcarrier allocation helps to reduce the effect of self-interference by exploiting the frequency diversity. A tradeoff between selfinterference reduction and performance gain justifies the selection of the codebook size and feedback scheme in LTE downlink environment.

\section{Acknowledgment}

The author would like to thank the National University of Malaysia for the financial support of this work, under the Grant scheme UKM-GGPM-ICT-032-2011. The author also would like to thank the anonymous reviewers for their valuable feedbacks.

\section{References}

[1] 3GPP Technical Specification 36.213, "Physical layer procedures (Release 8)," http://www.3gpp.org/.

[2] D. Gesbert, M. Shafi, D. S. Shiu, P. J. Smith, and A. Naguib, "From theory to practice: an overview of MIMO space-time coded wireless systems," IEEE Journal on Selected Areas in Communications, vol. 21, no. 3, pp. 281-302, 2003.

[3] H. Weingarten, Y. Steinberg, and S. Shamai, "The capacity region of the Gaussian multiple-input multiple-output broadcast channel," IEEE Transactions on Information Theory, vol. 52, no. 9, pp. 3936-3964, 2006. 
[4] S. Sanayei and A. Nosratinia, "Exploiting multiuser diversity with only 1-bit feedback," in Proceedings of the IEEE Wireless Communications and Networking Conference (WCNC '05), pp. 978-983, March 2005.

[5] D. Gesbert, M. Kountouris, R. W. Heath, C. Chan-Byoung Chae, and T. Sälzer, "Shifting the MIMO paradigm," IEEE Signal Processing Magazine, vol. 24, no. 5, pp. 36-46, 2007.

[6] D. J. Love, R. W. Heath, V. K. N. Lau, D. Gesbert, B. D. Rao, and M. Andrews, "An overview of limited feedback in wireless communication systems," IEEE Journal on Selected Areas in Communications, vol. 26, no. 8, pp. 1341-1365, 2008.

[7] H. Rohling and R. Grünheid, "Cross layer considerations for an adaptive OFDM-based wireless communication system," Wireless Personal Communications, vol. 32, no. 1, pp. 43-57, 2005.

[8] Philip RI-062483, "Comparison between MU-MIMO codebook-based channel reporting techniques for L TE downlink," 3GPP TSG RAN WG I Meeting \#46bis, october 2006.

[9] D. Yang, L. L. Yang, and L. Hanzo, "DFT-based beamforming weight-vector codebook design for spatially correlated channels in the unitary precoding aided multiuser downlink," in Proceedings of the IEEE International Conference on Communications (ICC'10), pp. 1-5, May 2010.

[10] B. Mondal and R. W. Heath, "Channel adaptive quantization for limited feedback MIMO beamforming systems," IEEE Transactions on Signal Processing, vol. 54, no. 12, pp. 47174729, 2006.

[11] S. J. Kim, H. J. Kim, and K. B. Lee, "Multiuser MIMO Scheme for Enhanced 3GPP HSDPA," in Proceedigs of the 11th European Wireless Conference, February 2005.

[12] J. Jang and K. B. Lee, "Transmit power adaptation for multiuser OFDM systems," IEEE Journal on Selected Areas in Communications, vol. 21, no. 2, pp. 171-178, 2003.

[13] J. P. Kermoal, L. Schumacher, K. I. Pedersen, P. E. Mogensen, and F. Frederiksen, "A stochastic MIMO radio channel model with experimental validation," IEEE Journal on Selected Areas in Communications, vol. 20, no. 6, pp. 1211-1226, 2002.

[14] K. C. Beh, C. Han, M. Nicolaou, S. Armour, and A. Doufexi, "Power efficient MIMO techniques for 3GPP LTE and beyond," in Proceedings of the IEEE 70th Vehicular Technology Conference Fall (VTC '09), pp. 1-5, September 2009.

[15] J. Zhu, J. Liu, X. She, and L. Chen, "Investigation on precoding techniques in E-UTRA and proposed adaptive precoding scheme for MIMO systems," in Proceedings of the 14th AsiaPacific Conference on Communications (APCC '08), pp. 1-5, October 2008.

[16] N. Ravindran and N. Jindal, "Limited feedback-based block diagonalization for the MIMO broadcast channel," IEEE Journal on Selected Areas in Communications, vol. 26, no. 8, pp. 1473-1482, 2008.

[17] K. C. Beh, Resource allocation for the long term evolution (LTE) of $3 G$ [Ph.D. thesis], University of Bristol, Bristol, UK, 2009.

[18] E. Y. Kim and J. Chun, "Random beamforming in MIMO systems exploiting efficient multiuser diversity," in Proceedings of the IEEE 61st Vehicular Technology Conference (VTC '05), vol. 1, pp. 202-205, June 2005.

[19] D. Tse and P. Viswanath, Fundamental of Wireless Communication, Cambridge University Press, New York, NY, USA, 2005. 

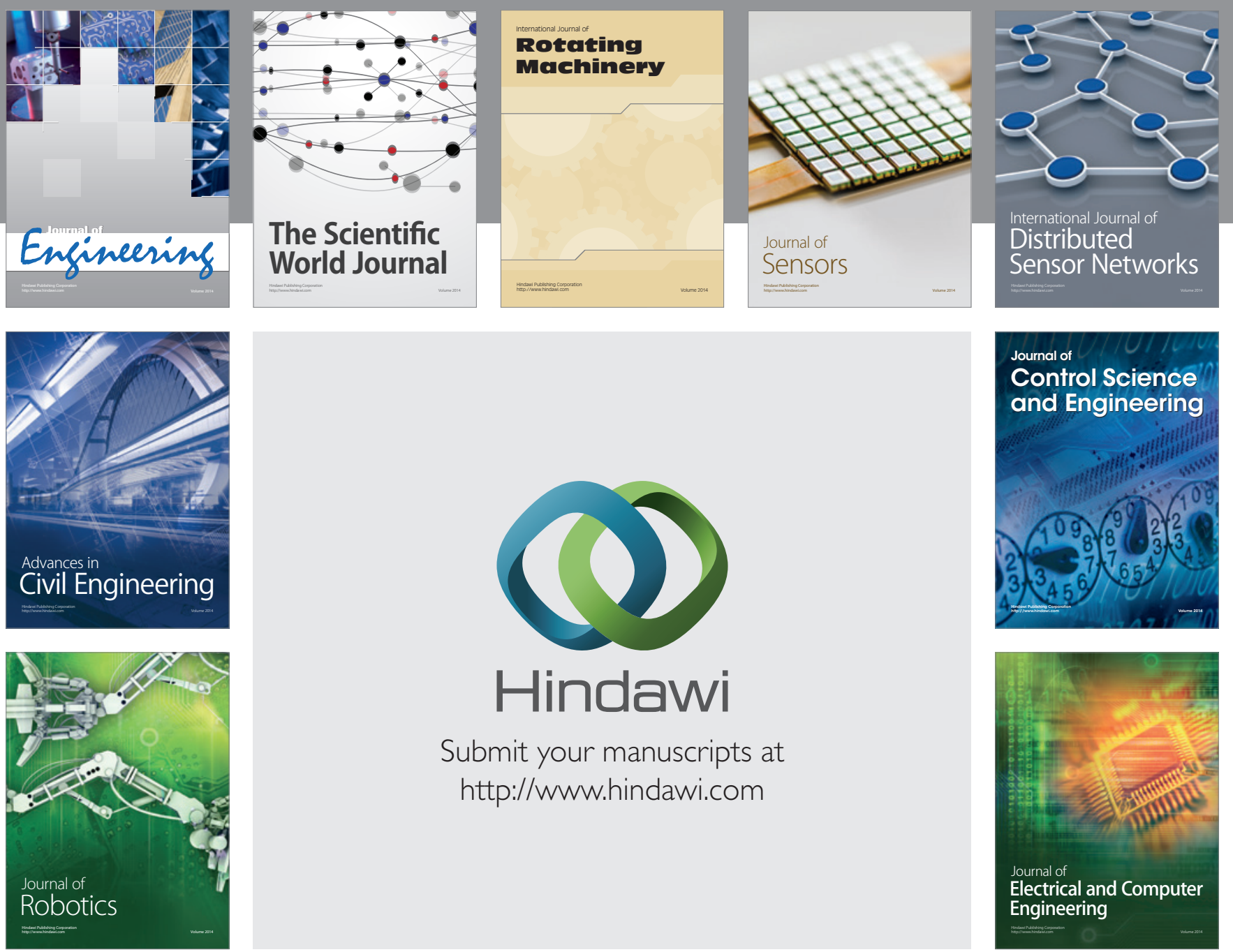

Submit your manuscripts at

http://www.hindawi.com
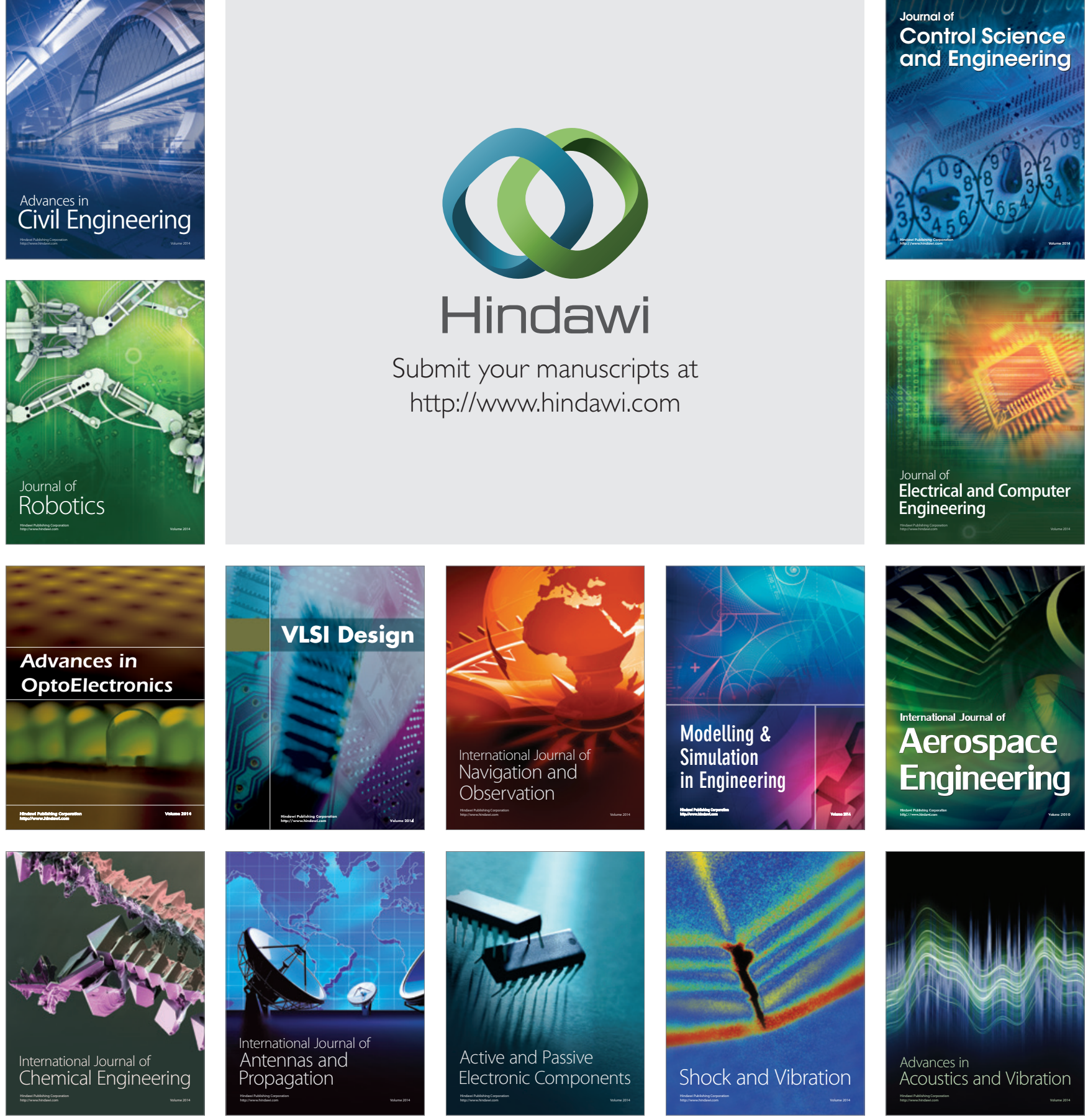